\title{
PHONON AND EXCITON AMALGAMATION - A CRITERION FOR TRUE SOLID SOLUTIONS: VIBRATIONS OF CHEMICALLY AND ISOTOPICALLY MIXED PARA-DIHALOBENZENE CRYSTALS*
}

\author{
James C. BELLOWS, Paras N. PRASAD \\ Department of Chemistry, State University of New York at Buffalo. \\ Buffalo, New York 14214, USA \\ and \\ Eric M. MONBERG and Raoul KOPELMAN \\ Department of Chemistry, University of Michigan, \\ Ann Arbor, Michigan 48109, USA
}

Received 5 December 1977

\begin{abstract}
The phonon and vibrathonal exciton spectra are suggested as criteria for true solid solutions of molecular crystals. The short range character of the interactions that determine both the phonon and exciton properties makes these bands ideal for distinguishing between truiy random mixed lattices and segregated microscopic domains. Raman studies of the chenicdily mixed $p$-dichlorobenzene $-p$-dibromobenzene crystal and the isotopically mixed $p$-dschlorobenzene-ha $-p$-dichlorobenzene- $d_{4}$ crystals illustrate the principles involved. All phonon bands (external molecular vibrations) of both the chemically and the isotopically mived crystals are in the amalgamation limit. Whle mass defects appear to determine the phonon frequency shifts in these mixed crystals, deviations from a virtual crystal model are observed and discussed. The low energy internal modes (vibrational excitons) are examined and found to be in the separated band limit. Treating the pure $p$-dichlorobenzene crystal as an isotopically mixed crystal due to the nutural abundance of the chlorine isotopes reveals that the chlorine stretch at $310 \mathrm{~cm}^{-1}$ is also in the separated band limit. All these mixed crystal systems are concluded to be substitutionally random on the molecular scale.
\end{abstract}

\section{Introduction}

The effect of substitutional disorder on molecular motions of organic solids is of considerable interest in understanding the energy transport and excitation relaxations in these systems $[1,2]$. Recent studies conducted in our laboratories have focussed on such investigations [3-5]. The effect of isotopic perturbation [3] and successive chemical perturbations on phonon bands $[4,5]$ in organic solids has been examined in detail. The present paper is an extension of our previous studies $[3,4]$ with the objective to examine both internal and external (phonon) vibrations in a substitutionally disordered solid and to compare the roles of isotopic defects with those of chemical defects.

\footnotetext{
* Supported in part by NSF Grant DMR77-00028 (Michigan).

* Alfred P. Sloan Research Fellow.
}

Our total experience with isotopically mixed cryetals $[2,3\}$ tells us that random substitutional disorder is the rule, and these alloys can be treated as "jdeal" solid solutions. For chemically mixed crystals, on the other hand, such a result is not as evident, but its possibility is of prime interest. Few methods are able to distinguish random substitution on the molecular scale from randomly distributed small aggregates of one or the other component. We note that when the size of such segregated domains is much smaller than the wavelength of light (say $\ll 4000 \AA$ ), neither ordinary optical nor diffraction methods (X-ray, neutron) will distinguish between random and semi-ordered forms of aggregation. On the other hand, in most molecular solids, and particularly those consisting of non-polar molecules, the phonon and vibrational exciton interactions are short-range and thus particularly sensitive to the organization of the aggregate on the molecular level. This sen- 
sitivity has been utilized in studies of excitons and phonons in crystals [2] as well as in the study of the structure and dynamics of liquids $[6]$. Thus it is through the study of the phonons and vibrational excitons in the mixed crystal that we hope to shed light upon the nature of the substitutional disorder in these systems.

For this investigation, both internal and external vibrations are examined in $\boldsymbol{p}$-dichlorobenzene- $\boldsymbol{h}_{\mathbf{4}}-\boldsymbol{p}$ dichlorobenzene- $d_{4}$ isotopically mixed crystals as well as in $p$-dichlorobenzene- $h_{4}-p$-dibromobenzene- $h_{4}$ chemically mixed crystals. We find that irrespective of the nature of the phonon motions, they exhihit the amalgamation limit behavior both in isotopically and chemically mixed crystals. The internal vibrations are usually found to be in the separated band limit [2] . Both results are consistent with compiete substitutional disorder, i.e., ideal mixing on the molecular scale.

\section{Experimental}

The $p-\mathrm{C}_{6} \mathrm{H}_{4} \mathrm{Cl}_{2}\left(\mathrm{DCB}-h_{4}\right)$ and $p-\mathrm{C}_{6} \mathrm{H}_{4} \mathrm{Br}_{2}\left(\mathrm{DBB}-h_{4}\right)$ were obtained from Aldrich Chemical $\mathrm{Co}$. and were zone refined before use. The $p-\mathrm{C}_{6} \mathrm{D}_{4} \mathrm{Cl}_{2}\left(\mathrm{DCB}-d_{4}\right)$ was obtained from Merck, Sharpe and Dohme. Mixed crystals were prepared by weight and grown from the melt by the Bridgman technique. Isotopically mixed crystals $\left(\mathrm{DCB}-h_{4}-\mathrm{DCB}-d_{4}\right)$ were assumed to grow at the nominal concentration. The chemically mixed crystals DCB- $h_{4}-\mathrm{DBB}-h_{4}$ were analyzed by gas chromatography to determine the actual concentration.

Raman spectra were obtained using a Spex model 14018 double monochromator with 1800 grooves $/ \mathrm{mm}$ holographic gratings. The spectral bandpass was 1 $\mathrm{cm}^{-1}$. Band positions were measured relative to the laser line and were reproducible to $0.3 \mathrm{~cm}^{-1}$. The absolute accuracy should be $\pm 0.5 \mathrm{~cm}^{-1}$ or better. Direct current detection was used. Excitation was provided by the $5145 \AA$ line of a Coherent Radiation argon ion laser (model CR-5) at $0.2 \mathrm{~W}$. Samples were cooled to $125 \mathrm{~K}$ in a stream of nitrogen vapor. Temperatures were measured with a chromel-constantan thermocouple and were controlled to $\pm 2 K$. Due to the differences in location and the glass envelope around the sample, the estimated accuracy of the temperature is $+5 \mathrm{~K},-0 \mathrm{~K}$.

\section{Results and discussion}

The Raman spectra in the phonon region of DCB$d_{4}, \mathrm{DCB} h_{4}, \mathrm{DBB}$ and two mixed crystals are shown in figs. 1 and 2 . Of the six expected phonons, five are resolved in all cases and the sixth is resolved in pure DBB (fig. 2). The sixth phonon has been observed [7] before in DBB and bromochlorobenzene, but it is not resolved in our mixed crystal studies. The mixed crystal spectra show that ail phonons are amalgamated. The amalgamation of phonons is expected for isotopically mixed crystals, but amalgamation is not necessarily expected for chemically mixed crystals. A previous study [4] has shown that the entire set of $p$-dihalobenzene mixed crystals with DCB exhibits amalgamation. Chemi-

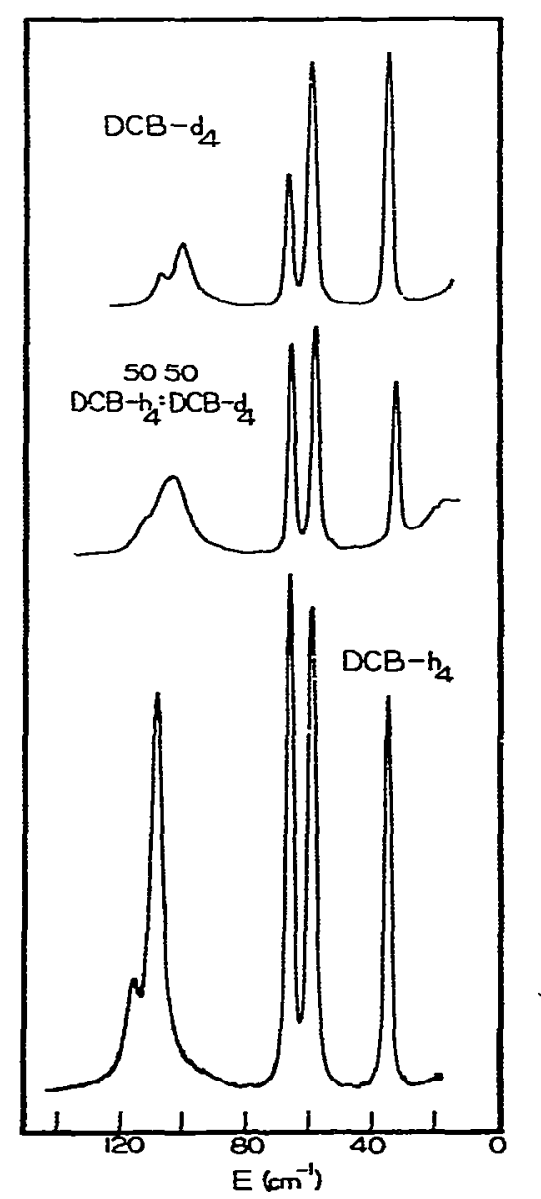

Fig. 1. Raman phonon spectra of pure and isotopically mixed crystals: $\mathrm{DCB}-d_{4}, 50$ mole \% $\mathrm{DCB}-h_{4}$ in $\mathrm{DCB}-d_{4}$, and DCB$h_{4}$ at $125 \mathrm{~K}$. 


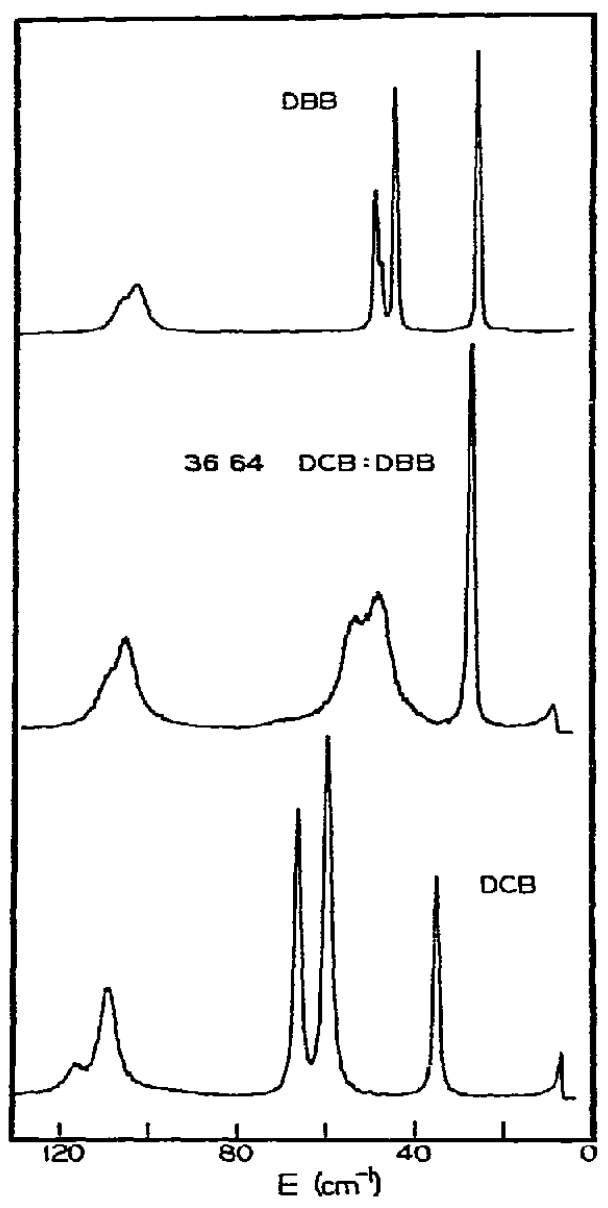

Fig. 2. Raman phonon spectra of pure and chemically mixed crystals: DBB, $36 \%$ mole DCB in DBB, and DCB at $125 \mathrm{~K}$.

cally mixed crystals of 1,4-dihalonaphthalenes also exhibit amalgamation [5]. There has been speculation [4] about the cause of amaigamation in these systems, but general conclusions require studies of many more systems.

The dependence of phonon frequency on composition is shown in fig. 3 . The band at $32 \mathrm{~cm}^{-1}$ has been previously [4] analyzed in terms of an average $T$-matrix approximation (ATA) for the chemical mixed crystal. The small linear shift in the isotopic mixed crystals can be understood in terms of the virtual crystal model $[2,4]$. The small change in frequency in the isotopic system is expected, since this phonon mode has been assigned to motions with large amplitưde on the halogens. The halogens dominate the mo-

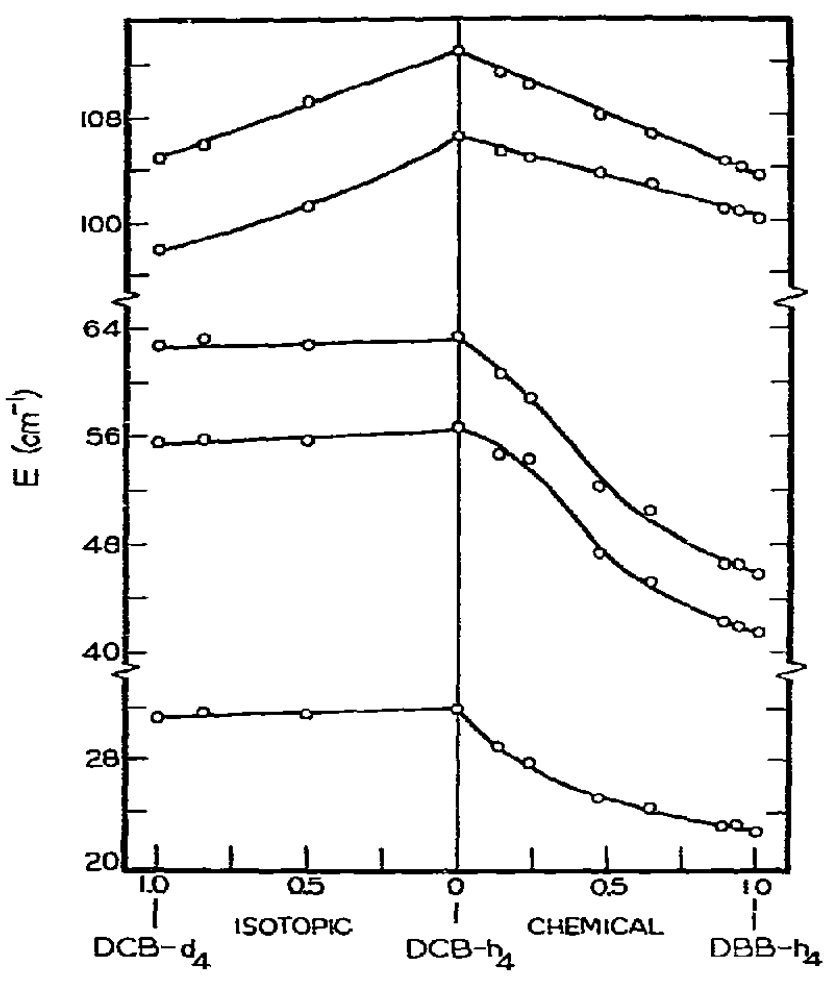

Fig. 3. Frequency dependence on composition in isotopically and chemically mixed crystals at $125 \mathrm{~K}$ : the phonon region.

ment of inertia and the addition of small mass incremerits at the hydrogen positions has little effect. The dominance by the halogen is again present in the case of the doublet at 57 and $63 \mathrm{~cm}^{-1}$. These phonons also show small isotope shift and large halogen shifts. The frequency dependence in the chemical mixed crystals can again be understood via an ATA model with a predominant mass defect [4]. The phonon doublet at 106 and $113 \mathrm{~cm}^{-1}$ (DCB- $\left.h_{4}\right)$ shows both large isotopic shift and large chemical shift. However, the frequency is essentially a linear function of composition. This fact indicates that the virtual crystal model may be applicable, al though in order to explain both the frequency shift and the line broadening one needs the ATA model [4] (see below) or the coherent potential model (CPA) [2]. The virtual crystal model indicates a relatively small perturbation and suggests again a, predominantly, mass defect. This phonon mode has been shown [8] to correspond chiefly to libration about the molecular axis passing through the two halogens (axis corresponding to $I_{\min }$ ). It is therefore expected 
that deuteration should have a large effect on this mode. However, calculations of the moments of inertia suggest that the observed difference in frequency between $\mathrm{DCB}-h_{4}$ and $\mathrm{DCB}-d_{4}$ is smaller by several percent than would be expected on the basis of libration about $I_{\min }$ exclusively. Mode mixing is required to reduce the difference in frequency. In addition, if the motion were exclusively about $I_{\min }$, there should be no shift in the chemically mixed crystals, provided there is no change in force constant. The presence of the large halogen shift is consistent with the mode mixing. Another important feature to be noted is the line broadening in the mixed crystals. A comparative study of fig. 1 and 2 shows that the doublet near $110 \mathrm{~cm}^{-1}$ $\left(\mathrm{DCB}-h_{4}\right)$ exhibits rather pronounced broadenings in the isotopic mixed crystal. On the other hand, the doublet near $60 \mathrm{~cm}^{-1}$ (DCB- $h_{4}$ ) shows relatively large broadenings in the chemically mixed crystal. This result is also consistent with the predictions of ATA [4] (or CPA $[2,9]$ ), which relate line broadening to the strength of the perturbation (mass defect in the present case).

Several internal vibrations show interesting crystalline effects. These are low lying modes in the region 250 to $350 \mathrm{~cm}^{-1}$. Fig. 4 shows a graphical representation of these internal vibrations in pure and mixed crystals. The doublets near $310 \mathrm{~cm}^{-1}$ and $350 \mathrm{~cm}^{-1}$, for $p$-dichlorobenezene, are due to a factor group (Davydov) splittings. The factor group splitting on the $350 \mathrm{~cm}^{-1}$ band of DCB- $h_{4}$ is $3 \mathrm{~cm}^{-1}$ in the pure crystal and decreases in both the chemical and isotopic mixed crystals until the components merge. This behavior is typical of the separated band limit $[2,10]$. The 310 $\mathrm{cm}^{-1}$ band of DCB- $h_{4}$ also shows separated band behavior in the isotopic mixed crystals where the magnitude of the splitting decreases as we increase the concentration of the $p-C_{6} D_{4} C l_{2}$. Furthermore, the reduction in the factor group splitting is also accompanied by a lowering of the frequencies. This usually indicates that the mean frequency of the factor group components is different from the frequency of the center of such vibrational exciton band $[11,2]$. In other words, the center of the exciton band shows an asymmetry. This can result if there is a contribution from excitation exchange interactions between translationally equivalent molecules. An alternative interpretation is that the static $D$-shift term is larger for $p-C_{6} \mathrm{H}_{4} \mathrm{Cl}_{2}$ than for $p-C_{6} D_{4} C l_{2}$. The corresponding band for $p-C_{6} D_{4} C l_{2}$

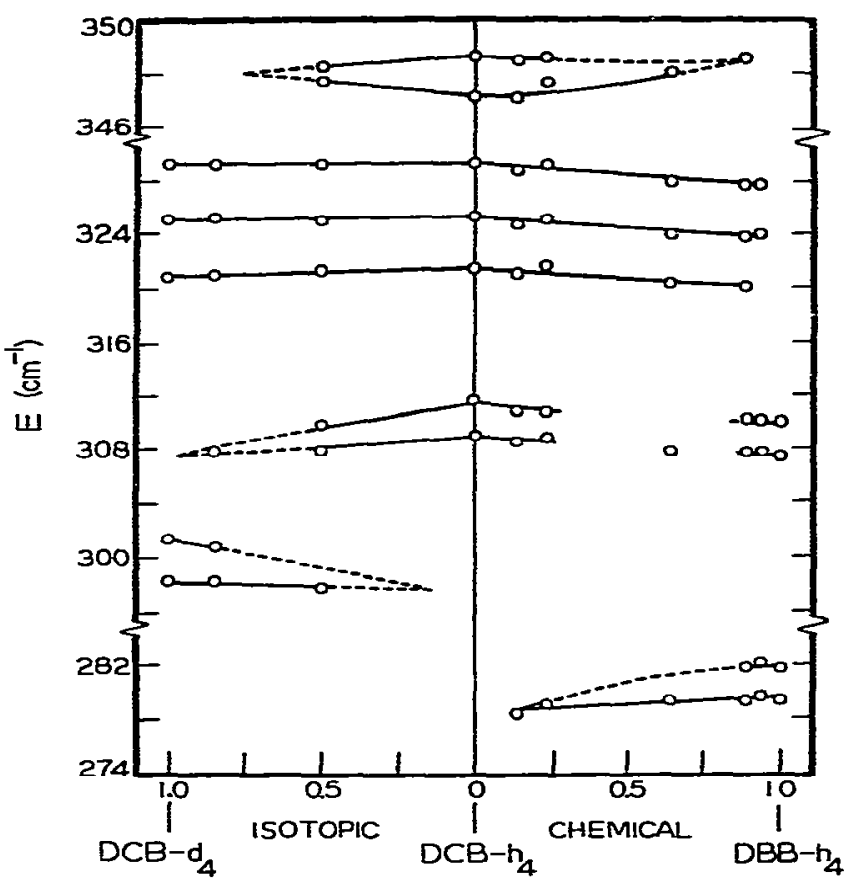

Fig. 4 Frequency dependence on composition in isotopically and chemically mixed crystals at $125 \mathrm{~K}$; internal vibrations in the 274 to $350 \mathrm{~cm}^{-1}$ region.

is the doublet around $300 \mathrm{~cm}^{-1}$ which also shows the separated band behavior. Here. again, in the isotopic mixed crystal the frequency shifts to lower values, i.e. the exciton band asymmetry is towards lower frequency. The vibrational exciton bands of both $p-\mathrm{C}_{6} \mathrm{H}_{4} \mathrm{Cl}_{2}$ and $p-C_{6} D_{4} C l_{2}$ thus show asymmetries in the same direction. This establishes that the asymmetry of the exciton band is due to the former explanation rather than due to a difference in $D$-shifts. In other words, translationally equivalent excitation exchange interactions are about as important as the interchange equivalent ones. This band also shows interesting behavior in the chemically mixed crystal. There are close lying bands in DBB (doublet near 308) but due to different kinds of vibrational modes. The possibility of amalgamation due to an intermolecular Fermi resonance [12] was carefully investigated. The results did not indicate amalgamation.

The triplet at $321,325,329 \mathrm{~cm}^{-1}$ shows separated band limit behavior in the chemically mixed crystals. Since the change in frequency on deuteration is only $0.5 \mathrm{~cm}^{-1}$ for the largest shift (321), we cannot say - 
whether the hydrogen isotope species are in an amalga mation limit or a separated band limit (or in-between). However, it is obvious that the chlorine isotope species (even in "pure" DCB- $h_{4}$ ) are in the separated band limit. The splitting (observed triplet) is a chlorine isotope effect. Three observations lead to this conclusion: First, the splitting is maintained in both the isotopic mixed crystals and the chemically mixed crystals, indicating that the splitting is not due to the factor group. Second, the frequencies are in the appropriate ratios for masses of 74,72 and 70 with identical force constants, and third, the intensities are always approximately $1: 6: 9$ as expected for an isctopic ratio of $1: 3$. This clear separation of the isotope splitting in the case of DCB is remarkable. It proves that there is little intermolecular coupling for this band. Indeed, the bandwidths must be less than $4 \mathrm{~cm}^{-1}$ or amalgamation would occur among the three species differing by $\mathrm{Cl}$ isotopes. This finding suggests that chlorine atons on adjacent molecules do not interact much as far as this mode is concerned. Since chlorine-chlorine interactions have been shown to dominate important aspects of the crystal structure [13] this result may be somewhat surprising. However, the explanation may be the fact that this mode has been shown [14] to be the symmetric chlorine stretch and only one of the $\mathrm{Cl}-$ $\mathrm{Cl}$ interactions has a large component in the direction of the motion

Finally, all the above data are consistent with a random site substitution model for DCB in DBB or vice versa. Any aggregation, even on a scale of $40 \AA$ or less, would result in very different spectroscopic behavior, i.e. a superposition of pure crystal (DCB and DBB) spectra*. Non-random disorder on an even finer scale

\footnotetext{
* Indeed, in the $p$-bromoiodobenzene-DCB system, segregation of phases does occur and gives rise to the superposition of two spectra. Such a spectrum is shown in fig. $1 \mathrm{C}$ of Bellows and Prasad [4]. However, in this case the two component spectra are those of solid solutions which presumably have random disorder.
}

would be expecied to result in significant deviations from both the amalgamated band limit and the separated band limit. There is also no evidence whatsoever of any phase transition driven by a change in concentration, unlike some other systems $[5]$.

\section{Acknowledgement}

Acknowledgement is made to the donors of the Petroleum Research Fund, administered by the American Chemical Society, for partial support of this research (at Buffalo).

\section{References}

[1] R.M. Huchstrasser and P.N. Pradad, in- Excited states, Vol. 1, ad. E.C. Lim (Academic Press, New York, 1974) p. 79.

[2] R. Kopelman, in: Excited states, Vol. 2, ed. E.C. Lim (Academic Press, New York, 1976) p 33.

[3] P.N. Prasad and R. Kopelman, J. Chem. Phys. 57 (1972) $863 ; 58$ (1973) 126.

[4] J.C. Bellows and P.N. Prasad, J. Chem. Phys. 60 (1977) 625.

[5] J.C. Bellows and P.N. Prasad, J. Chem. Phy's., to be published.

[6] R. le Sar and R. Kopelman, J. Chem. Phys. 66 (1977) 5035.

[7] N. Le Calvé, S. Parent and B. Pasquier, J. Raman Spectry. 2 (1974) 431.

[8] M. Ito, M. Suzuki and T. Yokoyama, in- Excitons, phonons and magnons in molecular crystals, ed. A.B. Zahlan (Cambridge Univ. Press, London, 1968) p. 1.

[9] H.-K. Hong and R. Kopelman, J. Chem. Phys. 58 (1973) $384,2557$.

[10] P.N. Prasad and R. Kopelman, J. Chem. Phys. 57 (1972) 856.

[11] E.R. Barnstein, S D. Colson, R. Kopelman and G.W. Robinson, J. Chem. Phys. 48 (1968) 5596.

[12] R. Kopelman, J. Chem. Phys. 44 (1966) 3547.

[13] P.N. Prasad and E.D. Stevens, J. Chem. Phys. 66 (1977) 862.

[14] A. Stojl1]kovic and D.M. Whiffen, Spectrochim. Acta 12 (1958) 47 